\title{
Application of Geographic Information Technology in Natural Disaster Emergency Management System
}

\author{
Fei Liu \\ Changjiang Institute of Technology, Wuhan Hubei, 430212, China
}

Keywords: Geographic Information Technology, Natural Disaster, Emergency Management System.

\begin{abstract}
This paper analyzes the characteristics of emergency management system of natural disasters and the content of management work, and on this basis, discusses the important role of geographic information technology in natural disasters emergency management system. Practice has proved that geographic information technology is helpful to nature Disaster management system to achieve dynamic, multi-dimensional development, to provide strong technical support for disaster management and emergency management.
\end{abstract}

\section{Introduction}

The problems of population increase, over-exploitation of resources, industrial pollution and other issues make the natural environment worse and worse, and natural disasters occur more and more frequently, which bring great harm to human life and property safety. Our country is one of the countries with the most serious natural disasters in the world; huge natural resources, environment and ecological pressure make our national natural disaster prevention contingency more complicated. Our government has always adhered to the principle of putting people first and always placed the safety of citizens in the first place in the protection of the life and property and incorporated disaster reduction in the planning system for social and economic development. In recent years, with the full implementation of the scientific concept of development, the construction of various disaster reduction mechanisms has been continuously strengthened, the capacity building for disaster reduction has been continuously improved and the cause of disaster reduction sustained and rapid development.

\section{Natural Disaster Emergency Management System}

There are many kinds of natural disasters in our country, and the occurrence of disasters is regional and seasonal, with high frequency and serious losses of disasters. The floods in the Yangtze River valley in 1998, the extraordinary drought in Chongqing in 2006 and the Wenchuan earthquake in 2008 all caused enormous losses to human lives and property. During the period when the global climate has been changing, the probability of occurrence of extreme weather and climate events gradually increases. Such conditions as anomalies in climate and uneven distribution of precipitation lead to increased natural disasters such as floods, droughts, fires, pests and diseases, and geological disasters such as flash floods, typhoons and debris flows Increasing possibilities, heavy natural disaster prevention tasks.

The major tasks of disaster reduction in China are to strengthen the building of hidden dangers and information management abilities of natural disasters, to fully understand the potential risks of natural disasters in various regions and to understand the disaster reduction abilities of various regions. According to the types, characteristics and frequency of natural disasters in different regions, Create a perfect database of natural disaster risk hidden dangers, and use the function of geographic information technology to draw a scientific and visual regional disaster risk map. We will create a natural disaster disaster statistics system and disaster reporting system, improve disaster information reporting mechanism, disaster information notification system and disaster information release platform, and improve the correct analysis and effective application of disaster information. 
Information management system can only provide current and previous data, therefore, a sound natural disaster emergency management system can not only be an information management system, but also has the function of early warning function of finding potential threats, comprehensive detection function and prediction of disaster development trend, making emergency decision-making functions. Natural disaster emergency management system can not only be a simple command system, but also should provide services for emergency management. It has the functions of predicting the occurrence of a disaster and correctly assessing the danger of sudden natural disasters, and has a dynamic and feasible accident handling plan. All emergency resources should be rationally allocated, and a more comprehensive and scientific information service should be provided for emergency management through actual combat manuals for interactive contingency plans so as to assist command and decision makers in formulating the best contingency plans.

Public safety science and technology is the core of normal operation of natural disaster emergency management system. Based on information technology, natural disaster emergency management system combining various hardware and software systems must include daily geography, emergency data management, disaster risk analysis, prediction and early warning, etc. Emergency management functions.

\section{Natural Disaster Emergency Management System Construction Mode and Their Own Characteristics}

At present, many departments and regions have tried natural emergency management system construction from many aspects and achieved fruitful results. Some emergency platforms with different focuses and characteristics have emerged in large numbers. According to different focuses, they can be summarized as the following modes:

\subsection{Alarm Unity Mode}

The establishment of this model is the earliest, the most prominent feature of this model is the a unified alarm and classification functions, this model effectively implements a alarm multi-functional, different police alarm acceptance even independent individuals, but also interoperability, police resources sharing also makes the police resources have also been fully demonstrated.

\subsection{A Combination of A Variety Communication Modes}

The most notable feature of this model is the effective combination of various communication methods such as wired communication and wireless communication, realizing centralized command and dispatch and increasing the convenience of command and dispatch. Some cities conducted digital trunking on the emergency command and communication system Construction.

\subsection{Video Conferencing Mode}

The most notable feature of this model is that based on the integration of video resources, the GZS system is widely used for surveillance in areas prone to natural disasters and decision-making and emergency response are conducted based on the video information seen.

\subsection{Information Management System Model}

This model of information submitted, classified, statistical function is very powerful, real-time access to and grasp of the current state of the data. This model attaches great importance to the construction of the database, the main role is to collect event-centric organization information, through the database for the service center to provide the required data information.

\subsection{Emergency Linkage Command Mode}

The main objective of this model is to achieve a rapid response. Various modes such as multi-police synergies will be integrated into an emergency management platform through various technologies such as communications, computers and the Internet. Direct leadership, command and coordination will be carried out by one department. 
These emergency management modes have given full play to their specific roles in facing different targets. The contingency management work has been deepened progressively. The society has increasingly higher requirements for disaster emergency response capabilities. All the above modes are not genuine natural disasters Management system. The construction of emergency management system for natural disasters must be closely integrated with the emergency management business process and follow the inherent laws and essential characteristics of various natural disasters to design emergency analysis and disposal functions and continuously optimize the alarm speed and improve the command and dispatch so as to fundamentally improve emergency response Disposal efficiency. The use of information technology is only a means of effectively using information technology to give full play to the role of information technology and effectiveness, but also need to resort to public safety technology.

\section{Natural Disaster Emergency Management System Based on Geographic Information Technology}

\subsection{Business Processes of Natural disaster emergency management system}

Emergency management of natural disasters is an extremely comprehensive system. The construction of the system involves a number of specialties. The business processes of a natural disaster emergency management system are as follows: incident $\rightarrow$ alarm $\rightarrow$ information reporting and handling $\rightarrow$ emergency start-up $\rightarrow$ rescue operation $\rightarrow$ state Control $\rightarrow$ emergency recovery $\rightarrow$ emergency termination $\rightarrow$ emergency summary $\rightarrow$ plan improvement or directly into the case base. If the information is not reported and handling links will be re-fed to the alarm information, if the situation is not controlled will be emergency reinforcements, and then will upgrade the response to continue to participate in the rescue operation. Emergency start includes information bulletin, the relevant personnel in place, experts in place, the preparation of emergency response teams, information collection and analysis. Rescue operations include on-site command members in place, relevant personnel and experts rushed to the scene, professional force support, engineering rescue, medical aid, evacuation and resettlement of people, environmental protection, on-site testing and public information communication. Emergency recovery includes on-site cleaning, de-alerting, aftermath handling and accident investigation.

\subsection{Functions of Natural Disaster Emergency Management System}

\subsubsection{Information Collection}

Natural disaster emergency management departments will be detected by the network to collect a variety of natural disasters, detailed information transmitted to the emergency management center.

\subsubsection{Information Performance}

Emergency management system will use intuitive and accurate information for the commander of command scheduling and decision support to provide technical and data support. Geographic information technology can be superimposed on the base map of geographic information natural disaster information layer, the emergency management of information content with the image of the spatial geography map out to emergency command and decision-making personnel more intuitively determine the occurrence of natural disasters, making more scientific decisions and allocating emergency resources in a rational manner all have positive help.

\subsubsection{Information Scheduling}

Decision-making personnel in the command center can formulate scientific decision-making and scheduling solutions through the information gathered in the system.

\subsubsection{Communication and Rescue Materials Scheduling}

Emergency rescue is an important part of emergency management. With emergency communication technology, human resources and material resources are reasonably scheduled. 


\subsubsection{Auxiliary Analysis and Decision-making}

Provide some feasible logistic analysis and statistics plan models and reference cases in the case base for the emergency rescue command, help the decision-making personnel in the command center to formulate more reasonable emergency decisions, and record the entire command and dispatch process Down, and scheduling process into a complete case, for intelligent management of emergency management to provide knowledge accumulation.

\subsubsection{Incident Tracking System}

Collecting the information of event site and responsible department, gathering various information contents into the management center through information annotation, using the functions of the central management system and displaying the information system of the emergent events graphically by using the geographic information as the background.

\subsubsection{Auxiliary Decision-making System}

Based on the knowledge path and meta-system data, the events and development trends of events are evaluated through the development of events, and the information system of the pre-set programs is demonstrated.

\subsection{System Design}

The design of natural disaster emergency management system based on geography information technology includes four main contents: system setting, map management, natural disaster incident management and auxiliary analysis. The system design uses C / S and B / S hybrid architecture system, set up a geospatial database in line with the system operation requirements, so that emergency management system can provide all kinds of data support for emergency management plan. Map management mainly includes map browsing, map switching, layer management, map drawing, query positioning, additional information and print output of these seven functions. Resource management is mainly the management of emergency agencies and emergency supplies. Aided analysis includes two functions: buffer analysis and network analysis. Buffer analysis can analyze the extent and extent of disaster impacts. By analyzing various types of disaster areas, we can clearly grasp the information such as affected population and provide data support for disaster assessment. Network analysis Fault analysis can be carried out to provide decision-making basis for disaster planning design and budget.

\section{The Role of Geographic Information Technology in Natural Disaster Emergency Management System}

First, it provides spatial data for the emergency management system of natural disasters and adds quick access and management functions of related attribute data. Natural disasters can meet the requirements of querying, updating, statistics and forecasting of natural disaster data better only if they have strong capability of handling a large amount of space and relevant attribute data rapidly and rapidly. The second is to provide a layered visualization of information functions, visualization of disaster information can be intuitive visual image of the system presented to the emergency command center. Thirdly, the interactive two-way query of the space and attribute data of the emergency management of natural disasters is realized, which helps the emergency management command center to find the disaster source information more quickly. Fourth, to promote the natural disaster emergency management system space and attribute data to achieve the integration of statistical analysis. Fifth, the spatial decision-making function of the natural disaster emergency management system has been added.

The combined use of geographic information technology and disaster analysis and prediction models in the emergency management system of natural disasters can provide timelier, comprehensive and scientific information support for emergency decision-making. The integration of geographic information technology and disastrous simulation model of disasters can build more powerful, more advanced, faster operation of the emergency management system.

Give full play to the role of geographic information technology in natural disaster emergency 
management system also need to solve the following aspects: First, we want to create a secure spatial database, and with the corresponding environmental database, test database combination; the second is to develop A set of perfect and intelligent data processing and analysis method; the third is to create a set of feasible disaster simulation analysis and evaluation mode; the fourth is to achieve a high degree of integration of geographic information technology and natural disaster emergency management system to reduce data exchange procedures and improve the natural Disaster emergency management system operating speed and analysis capabilities.

Natural disaster emergency management system must create a complete database to collect and collate various information contents such as spatial location, geographic information, disaster types, disaster characteristics and diffusion scope, effectively manage and systematically analyze the information through disaster emergency management system Deal with and improve the scientific nature of emergency response plans for natural disasters. In response to the actual needs of emergency response to natural disasters, the construction of emergency management system for natural disasters should change the actual functions of basic graphics and attribute information, multiple query and output attributes, and disaster hazard assessment functions.

\section{Conclusion}

The application of geographic information technology to natural disaster emergency management system can effectively realize the requirements of visualization of natural disaster emergency management space and improve the emergency response level and emergency management efficiency of natural disaster emergency management system. Natural disaster emergency management system combines the evaluation and analysis of GIS with the system simulation and forecasting model, expands the application range of GIS and improves the application level of the model in the management system. Natural disaster emergency management system, geographic information technology in data management, information visualization, to provide technical basis for decision-making have played a huge role. Multidimensional, real-time nature and comprehensive characteristics of emergency management system for natural disasters require that geographic information technologies should be organically integrated with other application systems in order to provide better comprehensive, timely and effective decision support for emergency response to natural disasters stand by.

\section{Acknowledgement}

This research was financially supported by the Research Based on GPS Indoor Location Technology.

\section{References}

[1] Ou Qijian. On Geographic Information Emergency Services Improvement, Side Rendering and Spatial Geographic Information, 2012,35.

[2] Wang Ting, CAO Junru, YUAN Xingming. Design of Urban Disaster Prevention and Mitigation Decision System Based on GIS, Shandong: Journal of Shandong University of Technology: Natural Science, 2012,01.

[3] Zhan Liang. DOA Based Emergency Management System GIS Platform for Key Technologies Research and Implementation, Chengdu University of Technology, 2014,05.

[4] GUO Hui, ZENG Wen-hao, HAN Xiao-jun. Research and Application of Back-up Emergency Support Service Information Platform, GEOGRAPHICAL INFORMATION, 2017,11.

[5] Ye Shan. Application of Spontaneous Geographic Information in Emergency Management of Natural Disasters, Office Automation, 2014, 07. 